\title{
Sonoanatomy for Anaesthetists
}

\author{
Edward Lin, Atul Gaur, Michael Jones, Aamer Ahmed. Cambridge University Press, \\ Cambridge, UK, 2012. \$76.95 CAD, 131 pages, ISBN 978-0-521-10666-5
}

\author{
Rob Tanzola, MD
}

Received: 30 April 2013/Accepted: 9 May 2013/Published online: 17 May 2013

(C) Canadian Anesthesiologists' Society 2013

Lin et al.'s Sonoanatomy for Anaesthetists is a timely publication given the increasing use of ultrasound in anesthesia practice. The authors have produced a text which could be a valuable resource for anesthesiology trainees and those with a particular interest in regional anesthesia. The authors' goal is to illustrate the relationships between surface anatomy, regional anatomy, and ultrasound scans for most of the common nerves and vessels that are subject to intervention by anesthesiologists. The book is not intended as a step-by-step guide for the performance of specific procedures, but rather as a description of the relevant sonoanatomy that can be applied to a variety of techniques. The authors endeavoured to achieve this by showing probe placement and the expected ultrasound finding for the nerve in focus.

The 131-page spiral-bound text begins with a brief general introductory chapter that describes the selection and types of ultrasound probes, the ultrasound appearance of different tissue, and needling and scanning techniques. Following this introduction, the text is divided into sections that focus on the neuroanatomy by anatomic area and are aptly titled: Upper limb, Lower limb, Back (focusing largely on ultrasound of the spine for neuraxial techniques), Head and Neck, and Abdomen. The final section, Vascular access, is dedicated to the ultrasound anatomical findings for blood vessels.

For each anatomic area, the relevant major nerves are examined in detail. For each nerve, the authors provide a short description of the anatomy, ideal probe placement and description of the relevant ultrasound findings, and tips

R. Tanzola, MD ( $)$

Kingston General Hospital, Queen's University, Kingston, ON, Canada

e-mail: tanzolar@kgh.kari.net for nerve blocks and their potential complications. Accompanying these short paragraphs, each nerve or vascular access site is illustrated with four figures: an anatomical figure, a clinical photograph showing the correct ultrasound probe position, the ultrasound scan, and a labelled illustration of the scan. These figures are the most useful feature of the text. The image quality of all the figures is excellent. Furthermore, the colour of the anatomic figures of each nerve and the diagram of its sensory distribution are exemplary and a helpful review. The photographs show the relevant anatomic regions and provide surface landmarks for probe placement and suggestions for the needle insertion site. The ultrasound scan images are clear with good contrast, and they are labelled to provide orientation and emphasize the relevant anatomic features. Lastly, the labelled colour diagram of the scan is displayed below the actual ultrasound scan and gives the reader a better appreciation of some of the subtleties of the ultrasound scan.

Although this is an excellent resource on the whole, there are some aspects that could be improved in future editions. One criticism concerns the labelling on some of the ultrasound scans in which the arrows used to point to the nerves are small equilateral triangles. Since the triangles do not indicate direction, they may confuse the reader and point them away from the structure of interest. A traditional arrow in these scans would be better to indicate the structure. The triangles are shown on only a minority of the scans, however, and any confusion is clarified when the scan and the corresponding colour diagram are used together. Given the focus on ultrasound anatomy, the scans themselves could be more prominent on the page. As ultrasound scanning is a dynamic exercise in localizing and following the course of structures, a welcome addition in future editions would be a video or 
online supplement of real-time scanning of the most common nerve blocks. One last criticism is disapproval of spiral-bound texts in general. This volume is intended as a practical book to take to the workplace and use in clinical practice, and the difficulty in navigating through it due to its spiral-bound nature takes away from its practicality.

These minor issues aside, Lin et al.'s Sonoanatomy for Anaesthetists is a reasonably priced comprehensive text. It is a visually appealing book with figures that convey a vast amount of information and accompanying text that is concise and complementary. It could be an excellent reference for practitioners interested in regional techniques as well as those looking for a refresher in the anatomy of peripheral nerves.

Competing interests None declared. 